occupational costs. Growing evidence links organizational risk factors to WRLBP.

Objective To examine the impact of work organization on WRLBP and mental health of Tunisian craftsmen.

Methods A cross-sectional study was conducted among the registered craftsmen of the governorate of Monastir (Tunisia) $(n=8526)$. The research was implemented on a representative sample, stratified by gender and craft activity. The survey included items related to health status, work organization, in addition to the Nordic Musculoskeletal and the Job Content Questionnaires.

Results The sample consisted of 368 craftsmen with a mean age of $42.7 \pm 8.5$ years and a sex-ratio of 0.88 . Work schedules were fixed with a mean duration of $8 \pm 2$ hours. Craftsmen had at least one break per day with a mean duration of $60 \pm 12$ minutes. Freedom in item design was found in $39 \%$ of cases. Room for manoeuvre was acceptable in 53\% of cases. Mutual help was possible in $40 \%$ of cases. Relationships with colleagues and superiors were 'good' in more than $80 \%$ of cases. According to the Karazek model, 13.6\% were in a Job Strain situation. WRLBP during the week preceding the survey were reported by $0.8 \%$ of the workers and $14.9 \%$ of them reported WRLBP during the last 12 months. Statistical analysis showed that WRLBP were less frequently reported by craftsmen with fewer hours of work $\left(\mathrm{p}<10^{-3}\right)$, those who had more than one break per day $\left(\mathrm{p}<10^{-3}\right)$ and the freedom of item design with non-monotonous production $\left(\mathrm{p}<10^{-3}\right)$. WRLBP was also less reported by workers non-constrained by an accelerated pace of work $\left(\mathrm{p}<10^{-3}\right)$, by a strict monitoring of work $(p=0.008)$, or by an increased sense of responsibility $\left(\mathrm{p}<10^{-3}\right)$.

Conclusion The risk factors for WRLBP are complex. Our research suggests that the role of organizational and psychosocial factors need to be better examined.

\section{P-458 GENDER INFLUENCE OF THE CLINICAL EXPRESSION AND SEVERITY OF COVID-19 INFECTION AMONG HEALTH CARE WORKERS}

${ }^{1}$ Lamia Bouzgarrou, Amen Moussa, Ben Afia Latifa, Amira Omrane, Safa Hsen, Touka Yahyaoui, Harrathi Chayma, Asma Kheder, Taoufik Khalfallah, Jihene Chili. ${ }^{1}$ University of Monastir, Tunisia

\subsection{6/OEM-2021-EPI.338}

Introduction Since the start of the COVID-19 epidemic, hospital staff have been massively affected in most countries, including Tunisia.

Objective This study aimed to examine the influence of the gender dimension on COVID-19 contamination expression among Tunisian hospital staff.

Methods Based on the COVID-19 register kept by the occupational medicine service and dedicated to hospital staff, data over 8 months were analyzed (September 2020-April 2021). COVID-19 screening was performed among all symptomatic hospital employees, or asymptomatic ones identified as close contact of a patient, colleague or relative confirmed positive for COVID-19. A nasypharyngeal swab was used for viral testing (reverse transcription polymerase chain reaction (RTPCR) and SARS-CoV-2 Rapid Antigen Test). Chest tomography was reserved for suspected cases with negative viral tests.
Results In total, 419 COVID-19 infections were confirmed by rt-PCR in $63.96 \%$, by rapid antigen testing in $33.65 \%$ and tomography in $2.39 \%$ of cases. Women represented $72.21 \%$ of contaminated staff, with average age of $21.75 \pm 9.78$ years but with no difference between gender groups. The department most affected was gynecology (43 cases), and nurses were the most affected category, especially among female staff $(p=0.016)$. In addition, fever $(p=0.012)$, neurological symptoms $(\mathrm{p}=0.049)$, such as aguesia $(\mathrm{p}=0.003)$, were more common in female workers. In contrast, digestive symptoms were more frequent in men $(p=0.0049)$, especially diarrhea $(p=0.00)$. In addition, chest tomography COVID-19 confirmation was significantly more common in men $(\mathrm{p}=0.012)$. Hospitalization was indicated in 16 cases, in intensive care in 2 cases with one case of death. No significant difference was noted between genders based on the severity of the COVID19 infection.

Conclusion Gender clinical difference of COVID-19 clinical expression was suggested by our results among health workers without impact on severity. These observations should be confirmed in larger studies.

\section{P-459 COPING STRATEGIES OF HOSPITAL NURSES IN THE COVID-19 PANDEMIC CONTEXT}

'Rosângela Aparecida de Sousa, Vivian Mininel. 'Universidade Federal de São Carlos, Brazil

\subsection{6/OEM-2021-EPI.339}

Introduction Occupational stress is one of the negative factors present in nursing work, especially in the hospital context; that factor was exasperated by the Covid-19 pandemic. Organizational strategies are very useful to reduce stress at work, individual coping behaviors can also contribute to the understanding of this factor.

Objective This study aims to understand coping strategies of hospital nurses with occupational stress in the Covid-19 pandemic context.

Method This is a qualitative, descriptive and exploratory study, which was developed at a university hospital located in the state of São Paulo, Brazil. The target population was male and female nurses who were employed in the research hospital. Data were collected through virtual individual interviews, based on a previously defined semi-structured questionnaire, and analyzed according to thematic content analysis. Intentional sampling was used and the participants were recruited until data saturation. The project was approved by study scenario and Ethics Committee for Research with Human Beings at Federal University of São Carlos; all ethical precepts were observed.

Partial Results Thirteen nurses participated in the data collection: 10 (76.92\%) women and $3(23.07 \%)$ men, aged between 31 and 55 years old. Working as a nurse: 1 (7.69\%) worked between 1 to 5 years, $3(23.07 \%)$ between 5.1 to 10 years, 6 (46.15\%) between 10.1 to 20 years and $3(23.07 \%)>20$ years. For the duration of work experience at the studied institution: $1(7.69 \%)<1$ year, $9(69.23 \%)$ between 1 to 5 years and $3(23.07 \%)$ between 5.1 to 10 years. It was expected, with the results of this research, to understand the coping strategies used by hospital nurses to cope with occupational stress in the current pandemic scenario of COVID-19. 